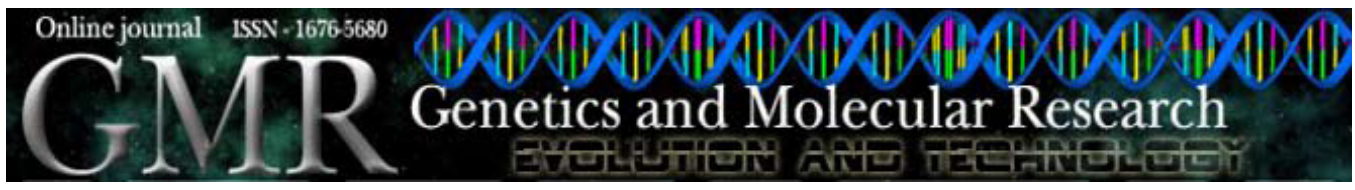

\title{
Increased ROS generation and SOD activity in heteroplasmic tissues of transmitochondrial mice with A3243G mitochondrial DNA mutation
}

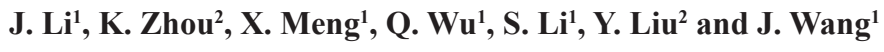 \\ ${ }^{1}$ Department of Laboratory Animal Science, Capital Medical University, \\ Beijing, China \\ ${ }^{2}$ Department of Biochemistry, Guangdong Medical College, Dongguan, China \\ Corresponding author: J. Wang \\ E-mail: wangju53@263.net
}

Genet. Mol. Res. 7 (4): 1054-1062 (2008)

Received June 9, 2008

Accepted June 30, 2008

Published October 14, 2008

\begin{abstract}
The mitochondrial A3243G tRNALeu(UUR) mutation associated with a variety of mitochondrial disorders results in a severe respiratory deficiency, an increase in reactive oxygen species (ROS) production and activities of anti-oxidative enzyme in vitro. However, the phenotypic implications of this mutation have not been described in vivo. Here, mitochondria carrying A3243G transition from the peripheral blood of diabetes mellitus patients were microinjected into zygotes. Influence of this mutation on mitochondrial respiratory enzyme activities, ROS generation, and antioxidative enzyme activities in the heteroplasmic tissues of transmitochondrial mice was evaluated. The chimeric mice exhibited a subtle impaired oxidative phosphorylation, reduced activity of complex I/IV, increased activity of superoxide dismutase, and in turn, enhanced ROS generation. Our results suggest that mitochondrial
\end{abstract}


A3243G mutation may be responsible for the high ROS production in vivo. Increased generation of ROS caused by mtDNA mutation may also play a role in the pathogenesis of the A3243G mutationassociated diseases.

Key words: Mitochondria; Mitochondrial DNA; A3243G mutation; Mitochondrial disorders; Transmitochondrial mice; Transmitochondrial

\section{INTRODUCTION}

Mitochondrial dysfunction caused by mitochondrial DNA (mtDNA) mutations is associated with a wide spectrum of human diseases. Although over 100 (Meulemans et al., 2006) types of different mtDNA mutations have been documented, point mutations in mitochondrial tRNA are particularly frequent (Kasraie et al., 2008). Among them, the mitochondrial A3243G tRNALeu(UUR) mutation is the most frequent (Urata et al., 1998) and has proven to be associated with various of single- or multi-organ syndromes, such as mitochondrial myopathy, encephalopathy, lactic acidosis, stroke-like episodes, etc. (Janssen et al., 2006; Mkaouar-Rebai et al., 2007). The occurrence of more than one mtDNA sequence variants in a single cell or individual is called as 'heteroplasmy'. The ratio of normal/mutant mtDNA determines the onset of mitochondrial diseases or disorders. When mutated mtDNA exceeds a minimum critical level or so-called "threshold", deleterious effects become possible (McKenzie et al., 2004; Gardner et al., 2007). Although heteroplasmy or threshold can partly explain the pathological phenotypes observed in patients, emerging evidence suggests no definite correlation between the clinical severity and the proportion of mtDNA mutation (Wang et al., 2002).

Apart from ATP generation, mitochondria are also the major source of reactive oxygen species (ROS) (Petrozzi et al., 2007). Emerging evidence from patients and in vitro studies suggests that mitochondrial dysfunction, either a primary (e.g., respiratory chain abnormalities) or a secondary (elevated ROS and control of apoptosis) event, plays an important role in the pathogenesis of mtDNA-based diseases (Ishikawa et al., 2005; Vives-Bauza et al., 2006; McKenzie et al., 2004, 2007). Patients carrying A3243G mutation exhibited an impaired oxidative phosphorylation with a deficient activity of respiratory chain complexes, increased ROS production, and significant induction of heme oxygenase-1 (Ishikawa et al., 2005). In vitro studies revealed the $\mathrm{A} 3243 \mathrm{G}$ mutation impaired RNA processing, aminoacylation, post-transcriptional tRNA modification, and translation (Mkaouar-Rebai et al., 2007). Transmitochondrial cybrids carrying A3243G showed decreased growth rate, lower oxygen consumption, defect complex I/IV, reduced electronic transfer chain activities and ATP synthesis, increased ROS production, decreased superoxide dismutase (SOD), and other antioxidant defense systems (Vives-Bauza et al., 2006; McKenzie et al., 2007). While A3243G mutation has been linked with mitochondrial dysfunction in vitro, the pathogenesis of A3243G mutation in animal models has not been investigated due to the limitation of transmitochondrial techniques.

In this study, the deleterious effects of $\mathrm{A} 3243 \mathrm{G}$ mutation on mitochondrial respiratory, ROS generation, and anti-oxidative enzymatic activities in the heteroplasmic tissues (hearts and brains) of transmitochondrial mice model were elucidated. Our results 
showed that the heteroplasmic tissues carrying A3243G mutation exhibited a subtle mitochondrial respiratory deficiency and a mild reduction of the complex I/IV enzymatic activities, indicating that the $\mathrm{A} 3243 \mathrm{G}$ mutation is responsible for the respiratory deficiency and the subsequent high ROS generation and increased SOD activity.

\section{MATERIAL AND METHODS}

\section{Animals}

$\mathrm{C} 57 \mathrm{BL} / 6 \mathrm{~J}$ female mice were used to generate zygotes via superovulation. ICR female mice were used to produce pseudo-pregnant mice. All animals were housed in groups under an artificial 12:12 lighting conditions with food and water available ad libitum and were maintained in a specific pathogen-free barrier facility. All procedures adhered to the Chinese Veterinary Medical Association Guide.

\section{Development of chimeric mice}

Mitochondria with A3243G mutation were isolated from the peripheral blood of diabetes mellitus patients following a method described by Pinkert et al. (1997). Briefly, tissue samples were suspended in $1 \mathrm{X}$ MS buffer $(210 \mathrm{mM}$ mannitol, $70 \mathrm{mM}$ sucrose, $5 \mathrm{mM}$ Tris$\mathrm{HCl}, \mathrm{pH}$ 7.5, $1 \mathrm{mM}$ EDTA, $\mathrm{pH}$ 7.5), homogenized for 20 times, and centrifuged at $1500 \mathrm{~g}$ for $10 \mathrm{~min}$. The supernatant was then spun at $9800 \mathrm{~g}$ for $10 \mathrm{~min}$ to pellet mitochondria and the pellet was washed with $150 \mathrm{mM} \mathrm{KCl}$ solution, recollected by centrifuging at $9800 \mathrm{~g}$ for 10 min, and resuspended in $40 \mu \mathrm{L} 150 \mathrm{mM} \mathrm{KCl}$. Microinjections were performed using inverted microscope (Pinkert et al., 1997).

All founders were genotyped by polymerase chain reaction (PCR) followed with ApaI digestion. The primers 5' CCT CCC TGT ACG AAA GGA CA 3' at position 3116-3135 and 5' GCC TAG GTT GAG GTT GAC CA 3' at position 3667-3647 were used for PCR (Zhang et al., 2005). The thermal profile consisted of denaturation of DNA at $95^{\circ} \mathrm{C}$ for $45 \mathrm{~s}$, annealing of DNA with primer at $56^{\circ} \mathrm{C}$ for $45 \mathrm{~s}$, and primer extension at $72^{\circ} \mathrm{C}$ for $56^{\circ} \mathrm{C}$. Amplification was usually done for 30 cycles and PCR-amplified DNA fragment was digested with the restriction enzyme ApaI (Promega). The ApaI-digested PCR products were then subjected to electrophoresis on a $1.5 \%$ agarose gel.

\section{Measurement of respiratory chain complex I-IV activities, ROS production, and antioxidant enzyme activities}

The respiratory chain complexes were assessed as described previously (Lionetti et al., 2004; Panov et al., 2005; Bonora et al., 2006; Meany et al., 2006; Long et al., 2007). For the assessment of ROS production, tissue samples were washed with PBS, digested with $0.5 \%$ trypsin and $0.1 \%$ collagenase for $40 \mathrm{~min}$, centrifuged at $600 \mathrm{rpm}$ for $5 \mathrm{~min}$ and resuspended in D-Hank's buffer. Approximately $1 \times 10^{6}$ cells were washed with PBS and resuspended in DHank's buffer. Forty microliters dichlorofluorescein diacetate (DCFH-DA; $2.5 \mathrm{mmol} / \mathrm{L}$ ) was added to each sample and incubated for $30 \mathrm{~min}$ at $37^{\circ} \mathrm{C}$ in the dark. Fluorimetric analysis was performed at $488 \mathrm{~nm}$ excitation and $535 \mathrm{~nm}$ emission. 
The antioxidant enzyme activities were measured using an SOD, glutathione peroxidase (GSH-Px), and catalase activity detection kit (Nanjing Jiancheng Bioengineer Institute, China) following the manufacturer instructions.

\section{Statistical analysis}

The results are reported as the mean $\pm \mathrm{SD}$. Statistical analysis was performed using the Student $t$-test.

\section{RESULTS}

\section{A3243G mutation in tissues of transmitochondrial mice}

To generate transmitochondrial mice carrying A3243G mutation, the mutated mitochondria isolated from diabetes mellitus patients were microinjected into C57BL/6J mice. Previous studies (Zhang et al., 2005) reported that the A3243G mutation, but not the wild-type mtDNA, can be recognized by ApaI. Therefore, restrict enzyme digestion by ApaI was performed to identify the positive mice. As shown in Figure 1, the digestion of mitochondria isolated from the transmitochondrial mice generated 423- and 130-bp fragments, respectively (Figure 1, lanes 2, 3 and 4). The mutant mtDNA was observed in the hearts and brains of two heteroplasmic mice (data not shown). However, due to the relatively poor transferring rate, only two male animals carrying the mutant mtDNA were obtained.

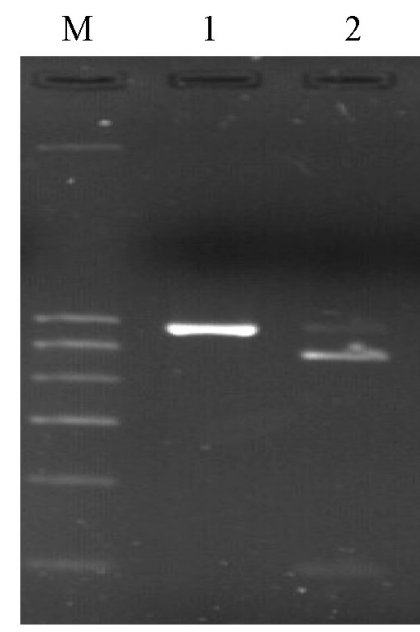

$553 \mathrm{bp}$

$423 \mathrm{bp}$

$553 \mathrm{bp}$

$423 \mathrm{bp}$

$130 \mathrm{bp}$

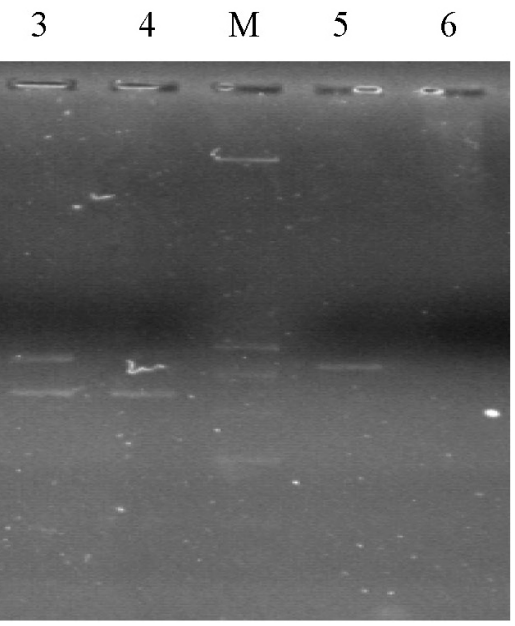

Figure 1. Genotype analysis of chimera mice by polymerase chain reaction (PCR) and ApaI digestion. mtDNA fragment amplified by PCR was digested with $A p a \mathrm{I}$ and size fractionated on agarose gel. Lanes 1 and $5=$ restriction pattern of control individual; lane $2=$ diabetes mellitus patient with $\mathrm{A} 3243 \mathrm{G}$ mutation; lanes 3 and $4=$ chimera mice carrying $\mathrm{A} 3243 \mathrm{G}$ mutation; lane $6=$ wild-type $\mathrm{C} 57 \mathrm{BL} / 6 \mathrm{~J}$ mouse. $\mathrm{M}=$ marker. 


\section{Chimera mice carrying A3243G mutation exhibited low mitochondrial complex I and IV activities}

To address if $\mathrm{A} 3243 \mathrm{G}$ mutation in the heteroplasmic hearts and brains influences mitochondrial respiratory chain function, the enzymatic activities of complex I (NADH: ubiquinone oxidoreductase), II (succinate:ubiquinone oxidoreductase), III (ubiquinol:cytochrome c oxidoreductase), and IV (cytochrome c oxidase) in chimeric mice $(\mathrm{N}=2)$ and age-matched wild-type $\mathrm{C} 57 \mathrm{BL} / 6 \mathrm{~J}$ mice $(\mathrm{N}=5)$ were assayed. As shown in Table 1 , in terms of respiratory enzymatic activities, no significant difference between chimeric mice and controls was observed (Table 1), probably due to small samples used. However, the heteroplasmic heart and brain tissues exhibited mild decrease of complex I and IV activities, while the activity of complex II and complex III remained unchanged (Table 1). As shown in Table 1, activity of complex I was reduced by $25 \%$ in the heteroplasmic hearts $(\mathrm{P}=0.072)$ and by $32 \%(\mathrm{P}=$ $0.106)$ in the heteroplasmic brains. Activity of complex IV was reduced by $27 \%$ in the heteroplasmic hearts $(\mathrm{P}=0.105)$ and by $40 \%$ in the brains $(\mathrm{P}=0.092)$, compared with control tissues. These data suggested that the $\mathrm{A} 3243 \mathrm{G}$ mutation in the heteroplasmic tissues caused a subtle defect in respiratory chain function, and the severity of mitochondrial respiratory deficiency in the brains was higher possibly owning to the higher proportion of mutant mtDNA. The finding of impaired respiratory function in the chimeric mice is in agreement with previous reports that the primary consequence of this mutation is a reduction in combined complex I/IV activities (Ishikawa et al., 2005; McKenzie et al., 2004, 2007).

\begin{tabular}{|c|c|c|c|c|c|c|c|c|}
\hline \multirow[t]{2}{*}{ Group } & \multicolumn{4}{|c|}{ Heart } & \multicolumn{4}{|c|}{ Brain } \\
\hline & Complex I & Complex II & Complex III & Complex IV & Complex I & Complex II & Complex III & Complex IV \\
\hline $\begin{array}{l}\text { C57/BL6J } \\
(\mathrm{N}=5)\end{array}$ & $33.91 \pm 3.99$ & $72.82 \pm 14.26$ & $1368 \pm 143$ & $3595 \pm 624$ & $23.19 \pm 4.73$ & $72.17 \pm 11.4$ & $1323 \pm 115$ & $1911 \pm 453$ \\
\hline $\begin{array}{l}\text { Chimera } \\
\text { mice }(\mathrm{N}=2)\end{array}$ & $25.28 \pm 6.21$ & $82.69 \pm 4.15$ & $1383 \pm 108$ & $2628 \pm 397$ & $15.82 \pm 4.84$ & $78.03 \pm 3.30$ & $1321 \pm 51$ & $1151 \pm 359$ \\
\hline$P$ value & 0.072 & 0.402 & 0.907 & 0.105 & 0.106 & 0.527 & 0.986 & 0.092 \\
\hline
\end{tabular}

Values are reported as $\mathrm{nmol} \cdot \mathrm{min}^{-1} \cdot \mathrm{mg}^{-1}$ Pro.

\section{Chimeric mice carrying A3243G mutation exhibited increased ROS production and anti-oxidative enzymatic activities}

We next asked if A3243G mutation influences ROS synthesis in vivo. To this end, ROS levels, SOD, GSH-Px, and catalase activities in the heteroplasmic heart and brain tissues were detected. As shown in Table 2, ROS level increased 14\% (P = 0.139) in the heteroplasmic heart and $18 \%(\mathrm{P}=0.059)$ in the heteroplasmic brain tissues, compared with controls. At the same time, total SOD activity in the heteroplasmic heart and brain tissues increased by $18 \%(\mathrm{P}=0.093)$ and $19 \%(\mathrm{P}=0.114)$, respectively (Table 3$)$. To our surprise, the GSH-Px and catalase activities remained unchanged (Table 3). Taken together, these data indicate that the A3243G mtDNA mutation increases ROS generation and total SOD activity in the heteroplasmic tissues. 


\begin{tabular}{lcc} 
Table 2. Reactive oxygen species (ROS) levels in the heteroplasmic tissues of chimera mice. \\
\hline Group & ROS production (DCF FI) \\
\cline { 2 - 3 } & Heart & $51.95 \pm 3.43$ \\
\hline C57/BL6J $(\mathrm{N}=5)$ & $53.56 \pm 4.13$ & $61.24 \pm 7.52$ \\
Chimera mice $(\mathrm{N}=2)$ & $60.91 \pm 7.55$ & 0.059 \\
P value & 0.139 & Brain \\
\hline Values are reported & & 0.139
\end{tabular}

Values are reported as fluorescence intensity $\cdot 1 \times 10^{6}$ cells $^{-1}$. DCF FI $=$ dichlorofluorescein fluorescence intensity.

\begin{tabular}{|c|c|c|c|c|c|c|}
\hline \multirow[t]{2}{*}{ Group } & \multicolumn{2}{|c|}{ SOD } & \multicolumn{2}{|c|}{ GSH-Px } & \multicolumn{2}{|c|}{ Catalase } \\
\hline & Hearts & Brains & Hearts & Brains & Hearts & Brains \\
\hline $\begin{array}{l}\text { C57/BL6J } \\
(\mathrm{N}=5)\end{array}$ & $49.04 \pm 6.28$ & $117.53 \pm 11.57$ & $22.52 \pm 6.17$ & $29.11 \pm 5.39$ & $3.10 \pm 0.59$ & $4.77 \pm 0.41$ \\
\hline $\begin{array}{l}\text { Chimera } \\
\text { mice }(\mathrm{N}=2)\end{array}$ & $58.38 \pm 3.63$ & $138.91 \pm 14.95$ & $21.67 \pm 1.82$ & $25.58 \pm 2.79$ & $3.34 \pm 0.18$ & $5.03 \pm 0.11$ \\
\hline$P$ value & 0.114 & 0.093 & 0.863 & 0.435 & 0.613 & 0.43 \\
\hline
\end{tabular}

Values are reported as $\mathrm{U} \cdot \mathrm{mg} \mathrm{Pro}^{-1} \cdot \mathrm{min}^{-1}$. SOD $=$ superoxide dismutase; GSH-Px $=$ glutathione peroxidase.

\section{DISCUSSION}

mtDNA mutations cause a wide range of clinical phenotypes characterized by mitochondrial respiratory chain dysfunctions. However, it still remains controversial whether mutated mtDNA would contribute to ROS generation. Even though several previous studies have shown that mtDNA mutation is related to augmented ROS production (Lionetti et al., 2004; Petrozzi et al., 2007; Carelli et al., 2007), controversial results have also been reported. For example, it has been shown that mice with mutant mtDNA do not exhibit increased ROS levels in spite of carrying a high mutational burden (Meissner, 2007). Another report has demonstrated that mutant cybrids carrying A3243G mtDNA from a patient suffering from MELAS have reduced activity of cytochrome c oxidase significantly, lower ATP level and decreased mitochondrial membrane potential, but the endogenous levels of ROS are very similar in all cybrids regardless of whether they carry the mtDNA defects or not (Sandhu et al., 2005). Therefore, up to date the interplay between A3243G mtDNA mutation and ROS has not been fully clarified.

We reported here that $\mathrm{A} 3243 \mathrm{G}$ mutation is responsible for the impaired respiration enzymatic activities, increased ROS generation and SOD activity in the heteroplastic tissues of transmitochondrial mouse model. These results strongly suggest that A3243G mtDNA mutation is linked with the disorder of ATP and ROS generation in vivo. Therefore, the mitochondrial dysfunction is a primary event in the A3243G mutation-based diseases' process. Dysfunction of the mitochondrial respiratory chain leading to decrease of energy production primarily affects tissues with high energy requirements and renders cells unable to adapt to conditions of reduced mitochondrial energy supply (Janssen et al., 2006). This might explain why the deleterious effects of A3243G mutation were detected both in brains and hearts, but not in other heteroplasmic tissues such as the kidneys and spleens. 
Mitochondria are not only a major source of ROS, but they also possess vigorous antioxidant defense including free radical scavengers and antioxidant enzymes such as SOD and GSH-Px (Hervouet et al., 2007). Besides the energy defect, A3243G mutation also causes ROS associated diseases' pathophysiology (Ishikawa et al., 2005; Vergani et al., 2007). For example, the A3243G mutation has been shown to increase total SOD and catalase activities in myoblasts (Rusanen et al., 2000). Increased ROS production was also reported in the endomyocardial tissues of a patient with A3243G mutation (Maechler and de Andrade, 2006). In agreement with the findings cited above, we observed enhanced ROS generation and SOD activity in the chimeric mouse models carrying A3243G mutation, but no change of GSH-Px and catalase activities was observed. SOD is an essential enzyme detoxifying superoxide radicals to hydrogen peroxide. Increase in SOD may result in the enhanced formation of hydrogen peroxide. This combined with unchanged in catalase activity could lead to increased hydrogen peroxide accumulation and thus inducing oxidative stress. The elevated ROS generation detected by DCFH-DA may be interpreted as increased oxidative stress in the heteroplasmic tissues. Therefore, ROS is a secondary event in the pathogenesis of A3243G mutation. The increase in ROS production in the heteroplasmic tissues is compatible with the decrease in activity of complex I, of which the flavin mononucleotide group has been proposed as the main physiologically relevant site of ROS production (Wei et al., 2004). The complex I inhibitor rotenone has been shown to increase $\mathrm{H}_{2} \mathrm{O}_{2}$ generation in prepared mouse mitochondria from the brain, heart, but not kidney (Beretta et al., 2006; Carelli et al., 2007). Comparative expression in warts and lymphocyte defect induces mitochondrial outgrowth as a consequence of increase ROS production (Bonora et al., 2006). It is therefore tempting to speculate that the clinic phenotypes of patients with A3243G mutation are in part due to stimulation of mitochondrial proliferation by ROS.

Taken together, these data support the "vicious" circle hypothesis. The A3243G mutations affect synthesis of respiratory chain subunits, resulting in respiratory dysfunction and enhanced ROS production. In turn, increased ROS production induces damage to lipids, proteins and DNA and enhances mtDNA mutations. This vicious cycle is proposed to cause an exponential increase of mtDNA mutations over time, leading to diseases (Trifunovic et al., 2005; Petrozzi et al., 2007; Long et al., 2007; Meissner, 2007).

\section{ACKNOWLEDGMENTS}

Research supported by Beijing science and technology grant. We are grateful to Zhiyun Quan and other members (Institute of Laboratory Animal Sciences, Chinese Academy of Medical Sciences and Peking Union Medical College) for technical assistance, to Yanfei Huang (Beijing Tongren Hospital, China) for clinical sample collection and to Dr. Qunzhou Zhang (Center for Craniofacial Molecular Biology, University of Southern California School of Dentistry, USA) and Prof. Wengong Wang (Department of Biochemistry, Peking University) for insightful comments.

\section{REFERENCES}

Beretta S, Wood JP, Derham B, Sala G, et al. (2006). Partial mitochondrial complex I inhibition induces oxidative damage and perturbs glutamate transport in primary retinal cultures. Relevance to leber hereditary optic neuropathy (LHON). Neurobiol. Dis. 24: 308-317. 
Bonora E, Porcelli AM, Gasparre G, Biondi A, et al. (2006). Defective oxidative phosphorylation in thyroid oncocytic carcinoma is associated with pathogenic mitochondrial DNA mutations affecting complexes I and III. Cancer Res. 66: 6087-6096.

Carelli V, La Morgia C, Iommarini L, Carroccia R, et al. (2007). Mitochondrial optic neuropathies: how two genomes may kill the same cell type? Biosci. Rep. 27: 173-184.

Gardner JL, Craven L, Turnbull DM and Taylor RW (2007). Experimental strategies towards treating mitochondrial DNA disorders. Biosci. Rep. 27: 139-150.

Hervouet E, Simonnet H and Godinot C (2007). Mitochondria and reactive oxygen species in renal cancer. Biochimie 89: 1080-1088.

Ishikawa K, Kimura S, Kobayashi A, Sato T, et al. (2005). Increased reactive oxygen species and anti-oxidative response in mitochondrial cardiomyopathy. Circ. J. 69: 617-620.

Janssen RJ, Nijtmans LG, van den Heuvel LP and Smeitink JA (2006). Mitochondrial complex I: structure, function and pathology. J. Inherit. Metab. Dis. 29: 499-515.

Kasraie S, Houshmand M, Banoei MM, Ahari SE, et al. (2008). Investigation of tRNA(Leu/Lys) and ATPase 6 genes mutations in Huntington's disease. Cell. Mol. Neurobiol. (PMID: 18386172; DOI: 10.1007).

Lionetti L, Crescenzo R, Mollica MP, Tasso R, et al. (2004). Modulation of hepatic mitochondrial energy efficiency with age. Cell. Mol. Life Sci. 61: 1366-1371.

Long J, Wang X, Gao H, Liu Z, et al. (2007). D-galactose toxicity in mice is associated with mitochondrial dysfunction: protecting effects of mitochondrial nutrient R-alpha-lipoic acid. Biogerontology 8: 373-381.

Maechler P and de Andrade PB (2006). Mitochondrial damages and the regulation of insulin secretion. Biochem. Soc. Trans. 34: 824-827.

McKenzie M, Liolitsa D and Hanna MG (2004). Mitochondrial disease: mutations and mechanisms. Neurochem. Res. 29: 589-600.

McKenzie M, Liolitsa D, Akinshina N, Campanella M, et al. (2007). Mitochondrial ND5 gene variation associated with encephalomyopathy and mitochondrial ATP consumption. J. Biol. Chem. 282: 36845-36852.

Meany DL, Poe BG, Navratil M, Moraes CT, et al. (2006). Superoxide released into the mitochondrial matrix. Free Radic. Biol. Med. 41: 950-959.

Meissner C (2007). Mutations of mitochondrial DNA - cause or consequence of the ageing process? Z. Gerontol. Geriatr. 40: 325-333.

Meulemans A, Seneca S, Lagae L, Lissens W, et al. (2006). A novel mitochondrial transfer RNA(Asn) mutation causing multiorgan failure. Arch. Neurol. 63: 1194-1198.

Mkaouar-Rebai E, Tlili A, Masmoudi S, Belguith N, et al. (2007). Mutational analysis of the mitochondrial tRNALeu(UUR) gene in Tunisian patients with mitochondrial diseases. Biochem. Biophys. Res. Commun. 355: 1031-1037.

Panov AV, Lund S and Greenamyre JT (2005). $\mathrm{Ca}^{2+}$-induced permeability transition in human lymphoblastoid cell mitochondria from normal and Huntington's disease individuals. Mol. Cell. Biochem. 269: 143-152.

Petrozzi L, Ricci G, Giglioli NJ, Siciliano G, et al. (2007). Mitochondria and neurodegeneration. Biosci. Rep. 27: 87-104.

Pinkert CA, Irwin MH, Johnson LW and Moffatt RJ (1997). Mitochondria transfer into mouse ova by microinjection. Transgenic Res. 6: 379-383.

Rusanen H, Majamaa K and Hassinen IE (2000). Increased activities of antioxidant enzymes and decreased ATP concentration in cultured myoblasts with the 3243A --> G mutation in mitochondrial DNA. Biochim. Biophys. Acta 1500: 10-16.

Sandhu JK, Sodja C, McRae K, Li Y, et al. (2005). Effects of nitric oxide donors on cybrids harbouring the mitochondrial myopathy, encephalopathy, lactic acidosis and stroke-like episodes (MELAS) A3243G mitochondrial DNA mutation. Biochem. J. 391: 191-202.

Trifunovic A, Hansson A, Wredenberg A, Rovio AT, et al. (2005). Somatic mtDNA mutations cause aging phenotypes without affecting reactive oxygen species production. Proc. Natl. Acad. Sci. U. S. A. 102: 17993-17998.

Urata M, Wakiyama M, Iwase M, Yoneda M, et al. (1998). New sensitive method for the detection of the A3243G mutation of human mitochondrial deoxyribonucleic acid in diabetes mellitus patients by ligation-mediated polymerase chain reaction. Clin. Chem. 44: 2088-2093.

Vergani L, Malena A, Sabatelli P, Loro E, et al. (2007). Cultured muscle cells display defects of mitochondrial myopathy ameliorated by anti-oxidants. Brain 130: 2715-2724.

Vives-Bauza C, Gonzalo R, Manfredi G, Garcia-Arumi E, et al. (2006). Enhanced ROS production and antioxidant defenses in cybrids harbouring mutations in mtDNA. Neurosci. Lett. 391: 136-141.

Wang Z, Liu S, Yang Y, Yuan Y, et al. (2002). Detection of A3243G point mutation in mitochondrial DNA from 10 cases of MELAS. Chin. Med. J. 115: 995-997.

Wei YM, Yu H, Zhao X and Bai H (2004). Effects of selenium dioxide on apoptosis, Bcl-2 and P53 expression, 
intracellular reactive oxygen species and calcium level in three human lung cancer cell line. Chinese-German J. Clin. Oncol. 3: 141-146.

Zhang Y, Wang ZX, Niu SL, Xu YF, et al. (2005). Phenotype heterogeneity associated with mitochondrial DNA A3243G mutation. Zhongguo Yi. Xuе. Ke. Xuе. Yuan Xue. Bao. 27: 77-80. 